\title{
A machine learning framework for genotyping the structural variations with copy number variant
}

Tian Zheng ${ }^{1}$, Xiaoyan Zhu ${ }^{1 *}$, Xuanping Zhang ${ }^{1}$, Zhongmeng Zhao ${ }^{1},{\text { Xin } \mathrm{Yi}^{2} \text {, Jiayin Wang }}^{1}$ and Hongle $\mathrm{Li}^{3^{*}}$

From 15th International Symposium on Bioinformatics Research and Applications (ISBRA '19)

Barcelona, Spain. 3-6 June 2019

\begin{abstract}
Background: Genotyping of structural variation is an important computational problem in next generation sequence data analysis. However, in cancer genomes, the copy number variant(CNV) often coexists with other types of structural variations which significantly reduces the accuracy of the existing genotype methods. The bias on sequencing coverage and variant allelic frequency can be observed on a CNV region, which leads to the genotyping approaches that misinterpret the heterozygote as a homozygote. Furthermore, other data signals such as split mapped read, abnormal read will also be misjudged because of the CNV. Therefore, genotyping the structural variations with CNV is a complicated computational problem which should consider multiple features and their interactions.
\end{abstract}

Methods: Here we proposed a computational method for genotyping indels in the CNV region, which introduced a machine learning framework to comprehensively incorporate a set of data features and their interactions. We extracted fifteen kinds of classification features as input and different from the traditional genotyping problem, here the structure of variant may fall into types of normal homozygote, homozygous variant, heterozygous variant without CNV, heterozygous variant with a CNV on the mutated haplotype, and heterozygous variant with a CNV on the wild haplotype. The Multiclass Relevance Vector Machine (M-RVM) was used as a machine learning framework combined with the distribution characteristics of the features.

Results: We applied the proposed method to both simulated and real data, and compared it with the existing popular softwares include Gindel, Facets, GATK, and also compared with other machine learning cores: Support Vector Machine, Lanrange-SVM with OVO multiple classification, Naïve Bayes and BP Neural Network. The results demonstrated that the proposed method outperforms others on accuracy, stability and efficiency.

Conclusion: This work shows that the genotyping of structural variations on the CNV region cannot be solved as a traditional genotyping problem. More features should be used to efficiently complete the five-category task. According to the result, the proposed method can be a practical algorithm to correct genotype structural variations (Continued on next page)

\footnotetext{
*Correspondence: zhu.xy@mail.xjtu.edu.cn; Ilh173@163.com

${ }^{1}$ School of Computer Science and Technology, Xi'an Jiaotong University, Xi'an

710049, China

${ }^{3}$ Department of Molecular Pathology, Henan Cancer Hospital, The Affiliated

Cancer Hospital of Zhengzhou University, Zhengzhou 450003, China

Full list of author information is available at the end of the article
}

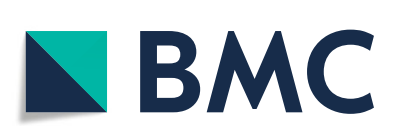

(c) The Author(s). 2020 Open Access This article is licensed under a Creative Commons Attribution 4.0 International License, which permits use, sharing, adaptation, distribution and reproduction in any medium or format, as long as you give appropriate credit to the original author(s) and the source, provide a link to the Creative Commons licence, and indicate if changes were made. The images or other third party material in this article are included in the article's Creative Commons licence, unless indicated otherwise in a credit line to the material. If material is not included in the article's Creative Commons licence and your intended use is not permitted by statutory regulation or exceeds the permitted use, you will need to obtain permission directly from the copyright holder. To view a copy of this licence, visit http://creativecommons.org/licenses/by/4.0/. The Creative Commons Public Domain Dedication waiver (http://creativecommons.org/publicdomain/zero/1.0/) applies to the data made available in this article, unless otherwise stated in a credit line to the data. 


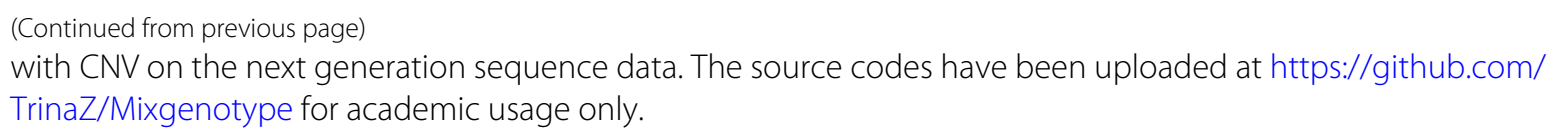

Keywords: Cancer genomics, NGS data analysis, Genotyping structural variation, Copy number variant, Multiclass relevance vector machine

\section{Background}

Structural variations(SVs) generally refer to cytogenetically visible and submicroscopic variants, including insertion, deletion, inversion, copy number variant and etc $[1,2]$. The genotype of SVs, also known as genotype analysis, is a technique to determine whether the structural variation is heterozygous or homozygous [3]. Obtaining the accurate genotypes of SVs can be widely used in downstream analysis, such as imputing genotypes [4], estimating genomic diversity [5], calculating linkage disequilibrium [6] and clinical practices including disease diagnosis [7], treatment management [8] and drug design [9].

Traditional methods used genomic chips to detect structural variations and their genotypes. In recent decades, with the development of the next generation sequence technology, sequencing data analysis has replaced the microarray. The existing methods often extract the data signals as features from the sequencing data and use these features to further estimate the genotypes. According to the strategies of incorporating the features, the existing methods can be divided into three categories, the first often rely on the overlapping points and breakpoints, such as Pindel-C [10]. The second category, include piCALL [11] and MATE-CELEVER [12], incorporate the features based on the Bayesian framework, and the third category, include Gindel [13] and CIGenotyper [14], adapt the machine learning models. Nevertheless, these methods do not take the effect of the copy number variant $(\mathrm{CNV})$ into account and suffer an accuracy loss for tumor sequencing data. CNV is a kind of common structural variant that widely exists in cancer genome, which plays an important role in cancerization [15], recurrence [16], metastasis [17], drug resistance [18], and is associated with clinical diagnosis [19] and antipsychotic drugs [18]. Recent studies reported that a CNV often combines with other structural variations [20,21], resulting in heterozygous variant being misjudged as homozygote, which seriously affects the accuracy of genotyping SVs on tumor data.

Specifically, to further investigate this computational problem, each human gene has two copies of the same haplotype and the CNV amplifies a one-sided signal, which causing the expression rate of the two gene copies deviated from 1:1 [22]. When a CNV appears on the haplotype harboring the variant, the number of reads in the mutated region presents multiple times more than that of the normal region, which may lead an existing method misclassifies a heterozygote to a homozygous variant, as shown by the red rectangle in Fig. 1. On the other hand, when a CNV appears on the wild haplotype, more reads mapped to the normal region will be observed and the signal of the variant side will be concealed, causing a heterozygous variant be misjudged as a non-variant homozygote, as shown by the blue rectangle in Fig. 1. Furthermore, these problems will be complicated by other data signals in cancer sequencing data. To name a few, all the tumor sample have the problem of purity and may cause the bias on data signals as shown by the black rectangle in Fig. 1, which is quite similar with the bias caused by CNV and may also be contributed by clonal structure. The tumor purity may change the variant allelic frequency (VAF) while may not contribute to the increase of coverage, if the purity causes the coverage of the normal hapolotper to be equal to the coverage of the mutated haplotype, a heterozygous variant with mutated haplotype $\mathrm{CNV}$ will be misjudged as a normal heterozygous variant, as shown by the red dotted rectangle in Fig. 1. Consequently, we have to further consider other data signals such as coverage, split mapped reads, read depth, extended read depth and their interactions to solve this problem. Moreover, recent studies reported that $\mathrm{CNV}$ is often combined with adjacent Single-Nucleotide Variants, which is one signal in detecting the CNV region and should also be considered. Other examples will not be repeated here, but it should be pointed out that other methods do not consider multiple features and their interactions may lead to computation exploration. It's inefficient to either consider single feature or use a Bayesian framework, while the machine learning framework is the most effective choice in this case with limited training samples.

In conclusion, the existing genotyping methods may suffer accuracy loss in processing the tumor sequencing data regardless the $\mathrm{CNV}$, and the genotyping problem should be considered comprehensively as shown in Fig. 1. The computation problem in this case is complicated into a five category problem which a structural variant may fall into one of the five category: normal homozygote $(\mathrm{N})$, homozygous variant (G1), heterozygous variant without CNV (G2), heterozygous variant with a CNV on the mutated haplotype (G3), and heterozygous variant with 


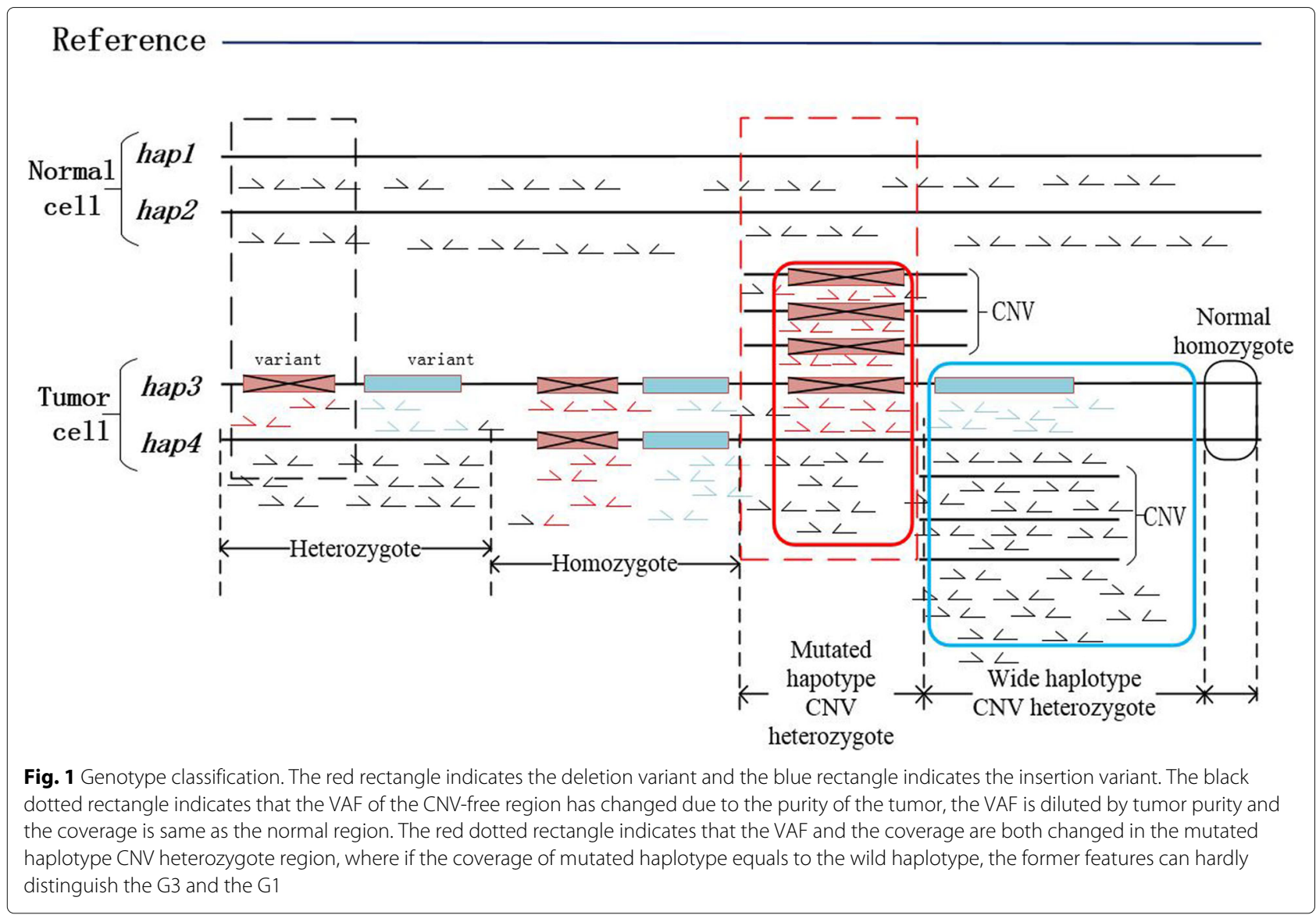

a CNV on the wild haplotype(G4). We proposed 15 features to train the model based on extensive research and chose the Multiclass Relevance Vector Machine(M-RVM) as the machine learning framework based on the data analysis. We applied the proposed method on both simulated and real data, compared it with the existing popular softwares include Gindel [13], Facets [23], GATK [24], and also compared with other machine learning cores, Support Vector Machine, Lanrange-SVM with OVO multiple classification, Nä̈ve Bayes and BP Neural Network. The results showed that the average of our method accuracy is $83 \%( \pm 2 \%)$ on simulated data and $88.2 \%( \pm 15 \%)$ on real data, while the classification accuracies of other methods are around $65 \%$ on simulated data and $75 \%$ on real data with more than $20 \%$ range at the same condition, which demonstrated that the proposed method outperforms others on accuracy, stability and efficiency.

\section{Methods}

\section{The genotype representations}

Considering the genotyping problem, we defined five classes of genotypes: normal homozygote $(\mathrm{N})$, homozygous variant (G1), heterozygous variant without CNV (G2), heterozygous variant with a $\mathrm{CNV}$ on the mutated haplotype (G3), and heterozygous variant with a CNV on the wild haplotype (G4). Each category is represented by vectors $[0,0,0,0,1]^{T},[0,0,0,1,0]^{T},[0,0,1,0,0]^{T}$, $[0,1,0,0,0]^{T},[1,0,0,0,0]^{T}$, respectively, which is one of the output vectors of M-RVM and the other output is their probability $\left[p_{N}, p_{G 1}, p_{G 2}, p_{G 3}, p_{G 4}\right]$, where $p_{N}+p_{G 1}+$ $p_{G 2}+p_{G 3}+p_{G 4}=1$ and $p_{N}, p_{G 1}, p_{G 2}, p_{G 3}, p_{G 4}$ represent the probabilities of the state $N, G 1, G 2, G 3$ and $G 4$, respectively. Note that the index set of the output vector is $I=\{N, G 1, G 2, G 3, G 4\}$, and M-RVM finally output the genotype with the highest probability.

\section{Input features extraction}

As mentioned above, to accurately classify genotypes has to incorporate multiple features which are extracted from the sequence data with the Standard Alignment/Map (SAM) or Binary Alignment/Map (BAM) format and list of candidate calls in the Variant Call Format (VCF). There are fifteen features considered in this approach as listed in Table 1. Details are discussed as follow.

Features based on the length of SV and the insert size relation The insert size is the length of template captured by the sequencer and the DNA fragments are expected to follow 
Table 1 Features description

\begin{tabular}{|c|c|c|}
\hline \multicolumn{3}{|c|}{ Description of Features } \\
\hline 1 & Abnormal read & $\begin{array}{l}\text { The number of abnormal insert size read } \\
\text { pairs }\end{array}$ \\
\hline 2 & Normal read & $\begin{array}{l}\text { The number of normal insert size read } \\
\text { pairs }\end{array}$ \\
\hline 3 & $\begin{array}{l}\text { Incompletely } \\
\text { mapped reads }\end{array}$ & $\begin{array}{l}\text { The number of incompletely mapped } \\
\text { read pairs }\end{array}$ \\
\hline 4 & Fully mapped read & $\begin{array}{l}\text { The number of read pairs that can fully } \\
\text { mapped to the reference }\end{array}$ \\
\hline 5 & Split mapped read & The number of split mapped reads \\
\hline 6 & Single mapped read & The number of single mapped reads \\
\hline 7 & Unmapped read & $\begin{array}{l}\text { The number of the mutated region } \\
\text { reads which comes from the Samtools }\end{array}$ \\
\hline 8 & Mapping qualities & $\begin{array}{l}\text { Sum of mapping qualities of anchor } \\
\text { reads }\end{array}$ \\
\hline 9 & Read depth & The read depth of the mutated region \\
\hline 10 & Weighted read depth & $\begin{array}{l}\text { The read depth of the mutated region } \\
\text { weighted by mapping qualities }\end{array}$ \\
\hline 11 & $\begin{array}{l}\text { Extended weighted } \\
\text { read depth }\end{array}$ & $\begin{array}{l}\text { Extended the weighted read depth to } \\
\text { up down } 100 \text { bps each }\end{array}$ \\
\hline 12 & Affected read & The number of affected reads \\
\hline 13 & Variant length & The length of structural variant \\
\hline 14 & Direction 1 & $\begin{array}{l}\text { The number of reads which clipping } \\
\text { from } 5^{\prime} \text { during initial mapping }\end{array}$ \\
\hline 15 & Direction 2 & $\begin{array}{l}\text { The number of reads which clipping } \\
\text { from 3' during initial mapping }\end{array}$ \\
\hline
\end{tabular}

the normal distribution around insert size in paired-end sequence [13]. We set $\mu$ as the mean library insert size and $\sigma$ as its standard deviation, which can be either specified by users or calculated from the given BAM/SAM file. We define the normal read as the read whose insert size located in the $\mu \pm 3 \sigma$ range, and position located in the mutated region. Accordingly, the read whose insert size deviates from normal distribution, exceeding the range of $\mu \pm 3 \sigma$, and position located in the mutated region is defined as the abnormal read. Consequently, we extract the numbers of normal reads and abnormal reads as two features as shown in Fig. 2.

When we consider the normal structural variant, the number of abnormal read is an important index to detect the indel. For example, large deletions may introduce the abnormal reads with extremely large insert size, and large insertions may introduce the abnormal reads with extremely short insert size. Furthermore, in the CNV region, this is more complicated. For a heterozygous variant without consideration other factors, the ratio of normal read to abnormal read is roughly close to $1: 1$, when a CNV exists in the mutated haplotype and the copy number equals to $n$, the ratio is close to $n: 1$, and when the CNV exists in the wild haplotype of a heterozygous variant, the ratio is close to $1: n$. Thus, different from the existing approach, the number of abnormal reads and normal reads should be considered at the same time. In addition, if the insertion is extremely longer than the insert size, the variant may not introduce the abnormal reads, while if the insertion is shorter than the insert size, the abnormal reads will be multiplied. Accordingly, the length of structural variant $(L)$ should also be considered as a feature, which can reflect the information about variant itself and indicate the difference in the relationship between the variant and the reads.

In practice, each row of the VCF file corresponds to a variant and indicates their matching positions respectively. Since the read length is $100 \mathrm{bps}$, we extract the second and the eighth columns, POS and End, from the VCF file, calculate the length of |POS-End| and record it as the $L$, and define the [POS-100, End +100$]$ interval as the mutated region for each variant; Then select the row of the BAM/SAM file which fourth column located in each mutated region and calculate the insert size of each read pairs. For each BAM/SAM file, calculate the mean and deviation of the insert size, and record the number of abnormal reads and normal reads for each structural variant.

\section{Features based on the alignment information}

We extract the numbers of incompletely mapped reads, split mapped reads, fully mapped reads, single mapped reads, unmapped reads, read directions, mapping qualities and affected reads as features based on the read alignment information.

Figure 3 lists five situations that a read located in a mutated region may fall into, if the reads are perfectly mapped to the reference, we extract the perfectly mapped read pair as the fully mapped read and the other as the single mapped read. Conversely, if the reads cannot be perfectly mapped to the reference, we define the read which one segment mapped to the reference and the rest cannot as the incompletely mapped read, define the read separated into two parts and each part mapped to the reference successfully as split mapped read, and define the rest cannot perfectly mapped read as unmapped read. For a region harboring a structural variant, the incompletely mapped reads may be caused if a non-homologous insertion mutation occurs. If a homologous deletion or insertion occurs, it may cause the split mapped reads. If the non-homologous insertion is extremely long and a whole read fall into the insertion fragment, the read may be counted as an unmapped read. Similar to the previous description, when there exists a CNV, the ratio that the SV caused reads to normal reads will be increased if the $\mathrm{CNV}$ happens in the mutated haploytpe, while the ratio will be deceased if the CNV happens in the wild haplotype.

Furthermore, the two reads in a read pair is usually in the opposite direction and some structural variations may 

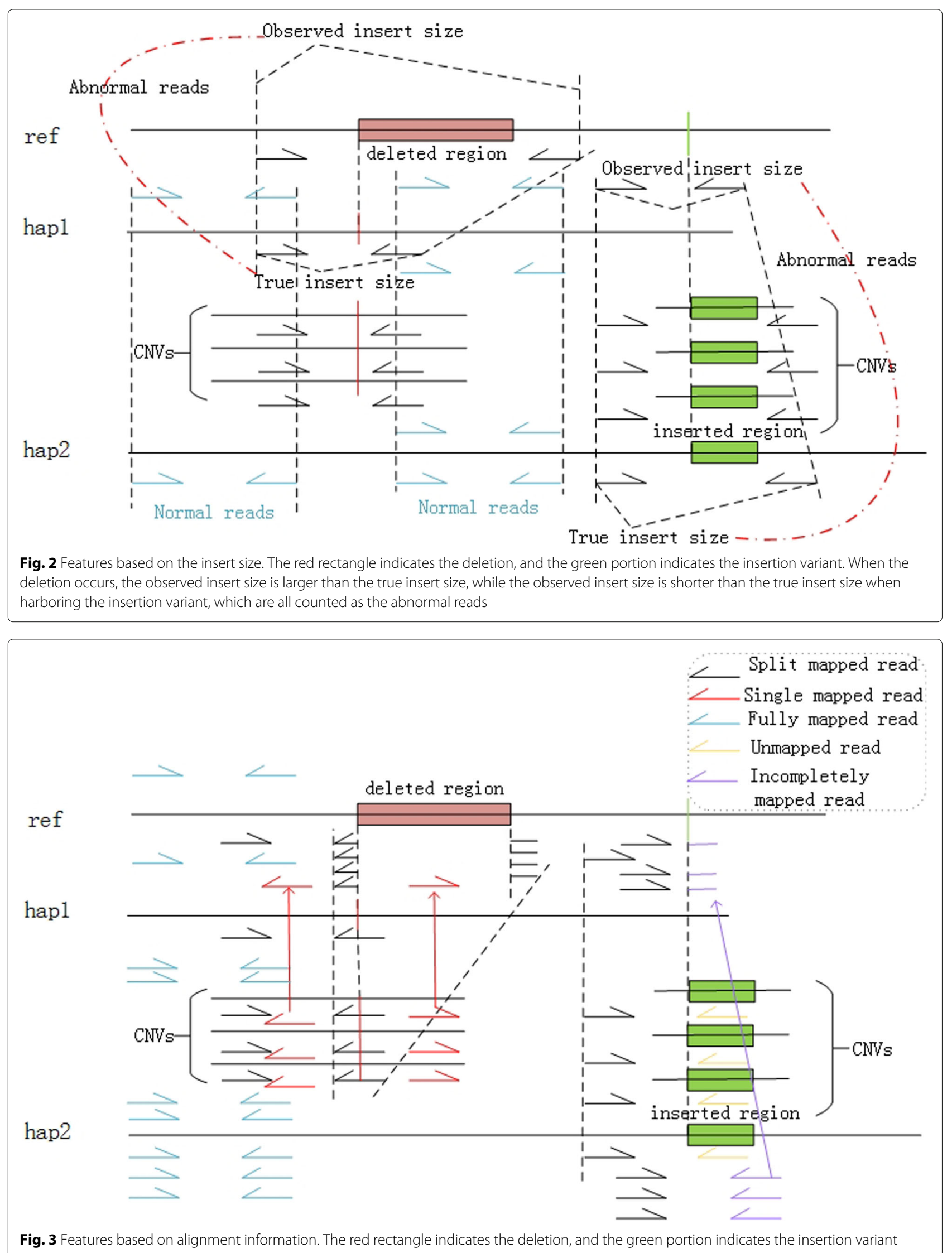
cause some reads to be reverse mapped to the reference, resulting the pair reads the same direction. If there are $\mathrm{CNV}$ and inversion, the reads harbor the inversion will show the same direction owing to the reverse complementarity, and the number of reads with same direction should not be ignored. Accordingly, we define the number of reads that clipping from $5^{\prime}$ during initial mapping and clipping from 3' as two features. Moreover, the mapping quality is a measure of the confidence that a read actually comes from the position it is aligned to by the mapping algorithm [25]. Because of the structural variation, the mapping quality of read pairs in mutated regions varies greatly from genotypes and copy numbers, and the sum of the mapping qualities of reads in mutated region can be used as a feature to identify genotypes.

Same as the former features, we first extract the mutated region for each structural variant, select the row in the BAM/SAM file which fourth column located in each mutated region and extract the CIGAR value of the row, which can fully reflect the alignment signals. We then count the read pairs whose CIGAR value is equal to "100M" as fully mapped reads, the single read whose CIGAR is equal to "100M" as single mapped read. On the other hand, we count the read whose CIGAR value is not equal to "100M" as incompletely mapped reads. Thirdly, for each pair of reads (every two rows), we count the number of read pairs whose fourth column POS value are one in and the other out of the mutated region as split mapped reads. Fourthly, we extract the reads which located in the mutated region and cannot match to the reference using the Samtools command "./samtools view -bS -bf -h **.f.bam $>{ }^{* *}$.sam" in the Linux terminal, so that the information file ${ }^{* *}$.f.bam file ${ }^{* * *}$ is the user-defined file name) of the unmapped reads is obtained. Fifthly, we count the number of reads which located in the mutated region and the second column FLAG equals to "83" as the direction 1 , count the number of reads which FLAG equals to "163" as the feature direction 2. Sixthly, we sum the corresponding fifth column MAPQ values of the reads that located in the mutated region in the BAM/SAM file for each SV, and record it as the value of Mapping qualities feature. The commands are all listed in the bat.sh file.

Last but not least, existing research found that there is a higher probability of base variation in the vicinity when structural variation occurs and different structural variations may lead to different probabilities of base variation [26]. Consequently, we extract the number of peripheral read pairs affected by structural variation as a feature and named it as the affected reads, which can reflect the existence characteristics of structural variation and facilitate the identification of multiple genotypes. We extract the second column and the eighth column, POS and End, from the VCF file, and set the [POS-length/10, End+length/10] as the variation vicinity range. We count the number of reads which fourth column POS value located in the variation vicinity range and recorded it as the affected reads value for each variant.

\section{Features based on the read depth}

Read depth refers to the number of reads mapped to a particular site or genomic region. Assuming that the sequence process is uniform, read depth follows a random (typically Poisson or modified Poisson) distribution [13] and the number of reads mapping to a genomic region is expected to be proportional to the ploidy that the region appears in the sequence sample. Compared with the normal region, the number of read depths in the mutated region will be reduced, while will be increased in the $\mathrm{CNV}$ region. Accordingly, the read depth may be a feature to distinguish five genotypes and we set the read depth(D) equals to the number of reads that located in the mutated region divide the $L$. Furthermore, we propose the weighted read depth as a new feature by weight the read depth with the coefficient $w_{i}$.

$$
w_{i}=\frac{Q_{i}}{Q_{\max }} .
$$

Where the $w_{i}$ refers to the coefficient, $Q_{i}$ refers to the $M A P Q$ value of each mapped read pair, $Q_{\max }$ equals to the highest MAPQ value of the read in one BAM/SAM file. And then the weighted read depth can be calculated as:

$$
W_{R D}=\frac{\sum_{i=0}^{n} w i}{L}
$$

The $n$ refers to the number of reads that in the mutated region of each variant.

We further propose the extended weighted read depth feature to avoid considering the mutated region only and to make full use of the difference between the mutated region and the normal region. Comparing with the weighted read depth, we expand the mutated region from [Pos - 100, END + 100] to [Pos - 200, END + 200], based on the relationship between the reads and the $L$. The expanded weighted read depth can reflect the genotype characteristics of multiple variants from another point of view.

\section{Framework selection rationale}

To select the suitable machine learning framework, we sampled a random $1 \mathrm{Mbps}$ region from the reference (version:hg19) and randomly planted 100 structural variants for each dataset. The type of the variants include insertion, deletion, inversion, complex indel and CNV. We created 20 candidates for each genotype and set the lengths of variations between $0.5 \sim 5 \mathrm{kbps}$, while the lengths of $\mathrm{CNVs}$ between $1 \sim 5 \mathrm{kbps}$. For each variant, we set an elevated region with $1000 \mathrm{bps}$ longer than its own length 
and set the regional mutation rate to 0.01 , some associated single nucleotide variants (SNVs) were planted in the preset elevated region and the background mutation rate was set to 0.0001 . About one fourth inserted fragments of the complex indel came from nearby regions [27]. We set the read length to $100 \mathrm{bps}$, the distribution of insert sizes to follow the normal distribution of the 500 bps mean and 15 bps standard deviation, and the sequence error rate of reads sampling was considered as 0.005 . We extracted the features and plotted them in Fig. 4, where the vertical axis represents the value of the features and the horizontal axis represents the structural variant calls. From 0 to 100, each of the 20 calls represent N, G1, G2, G3 and G4, respectively. The figure indicated that the type $\mathrm{N}$ can be distinguished from types G1-4, but types G1, G2, G3, G4 are difficult to classify. We tried a variety of machine learning models and found that the Multiclass Relevance Vector Machine (M-RVM) [28] works well for our datasets, and the five categories can be easily classified after the M-RVM kernel function transformation as shown in Fig. 5.

Compared with the popular methods and according to the description of the literatures, we suggest the M-RVM has the following advantages: 1. M-RVM adopts hierarchical Bayesian model structure and has the characteristics of flexible kernel function, overcoming the limitation that the selected kernel function must satisfy the Mercer condition, it is easier to find a suitable kernel function. 2. The membership probabilities of multiple classes output are realized by introducing Multi-probability likelihood functions. The M-RVM obtains probabilistic output and can directly output the prediction probability of each category.
3. M-RVM does not always depend on all feature data, which greatly reduces the calculation of kernel function, the test time and avoids over-fitting. 4.The M-RVM actually calculates the inner product of input vectors in feature space indirectly through the kernel mapping and does not need to be solved in high-dimensional space, which skillfully avoids the "Curse of Dimensionality" caused by the dimensions increase. We chose the M-RVM framework to deal with the linear and nonlinear datasets in higher dimension, and generated a more complex surface model in higher dimensional space according to data characteristics, so as to classify high-dimensional complex datasets non-linearly.

\section{Results}

\section{Generating simulated datasets and testing model parameters}

To evaluate the performance of the proposed method, we sampled a random 1 Mbps region from the reference (version:hg19), and then randomly planted 300 structural variants for each dataset. The type of the variants include insertion, deletion, inversion, complex indel and CNVs. Based on the distribution probability of $\mathrm{CNV}$, we created 60 Type $\mathrm{N}$ calls (normal homozygous genotype without variants), 80 Type G1 calls (homozygous variant without $\mathrm{CNV}$ ), 80 Type G2 candidates (heterozygous variant without $\mathrm{CNV}$ ), 50 Type $\mathrm{G} 3$ candidates (heterozygous variant with CNV occurs in mutated haplotype) and 30 Type G4 calls (CNV occur in wild haplotype heterozygote). Set the lengths of variations between $0.5 \sim 5 \mathrm{kbps}$, while the lengths of CNVs between $1 \sim 5$ kbps. For each variant, we set an elevated region with 1000 bps longer than its

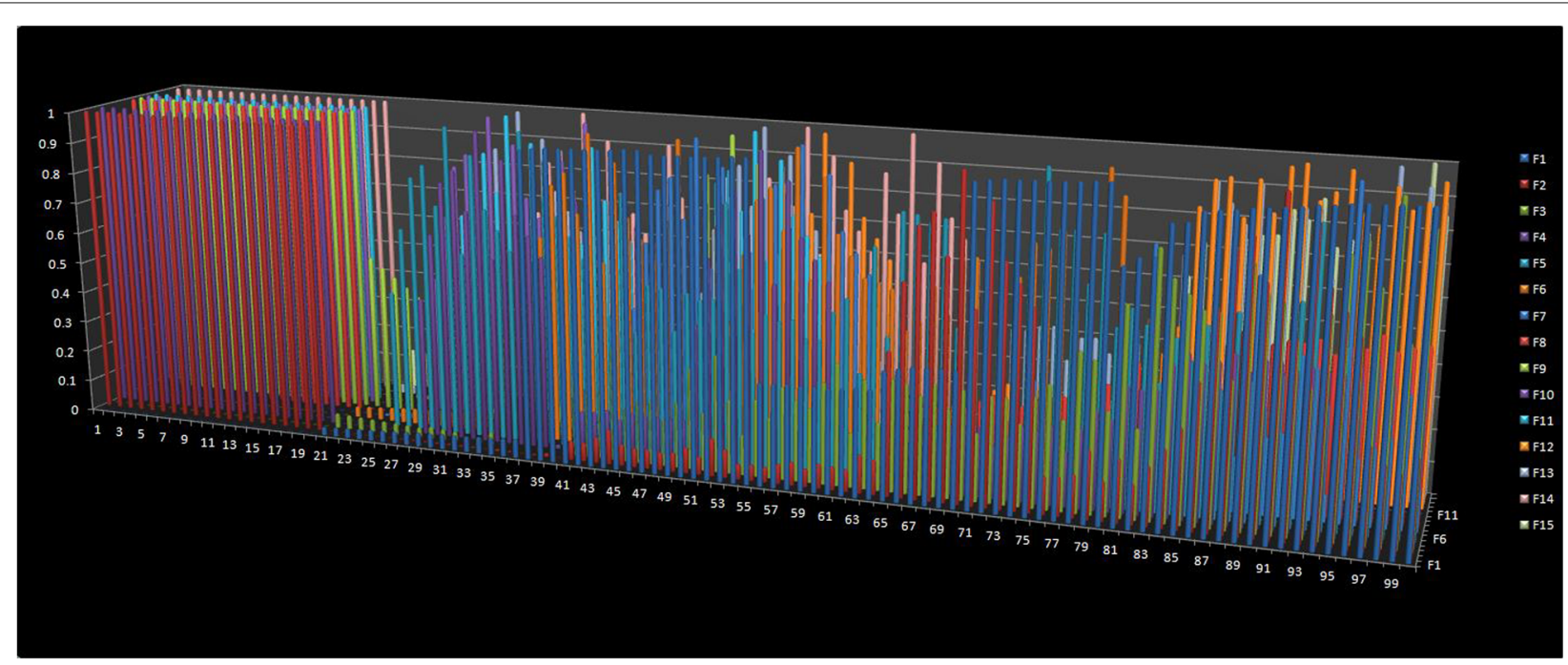

Fig. 4 The distributions of the features values. The vertical axis represents the value of the features and the horizontal axis represents the structural variant calls. From 0 to 100, every 20 calls represent N, G1, G2, G3 and G4, respectively. F1 to F15 follows the same order as listed in Table 1. The figure indicated that the type N can be distinguished from types G1-4, but types G1, G2, G3, G4 are difficult to classify 


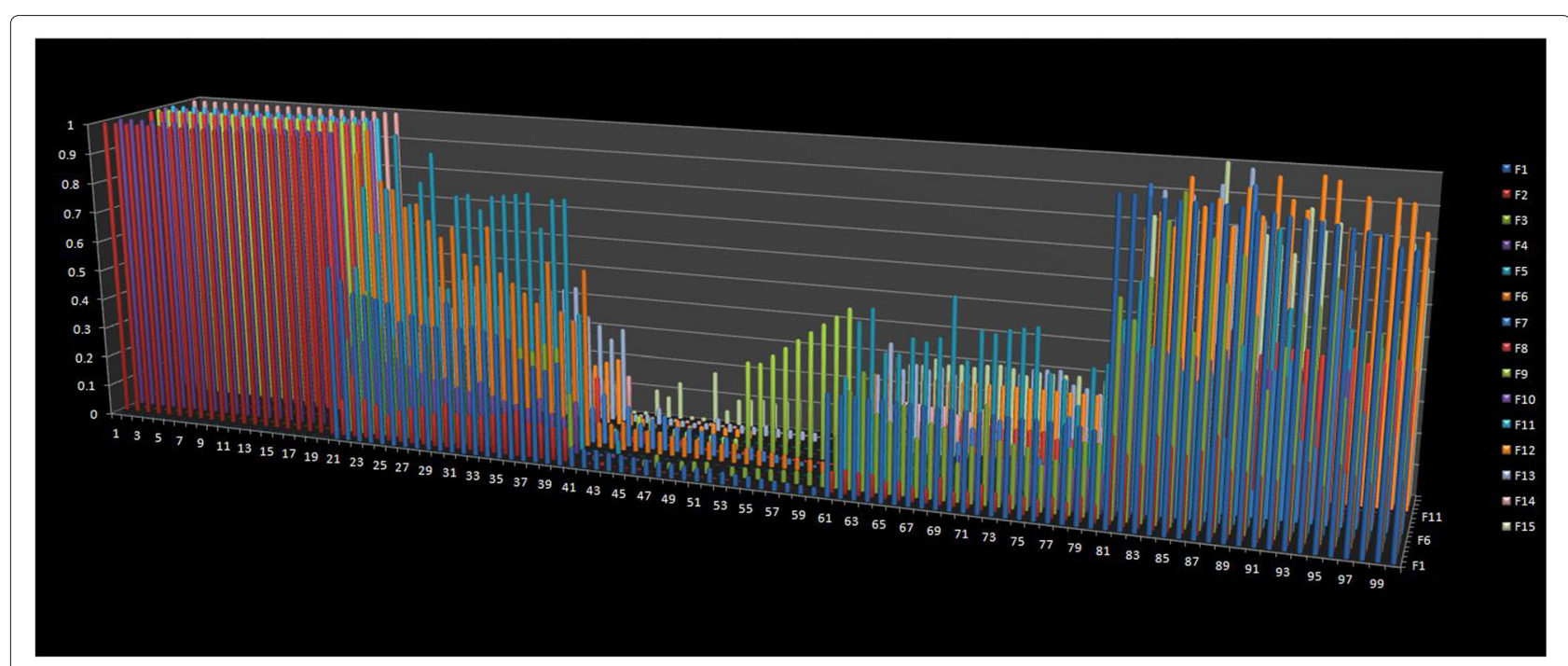

Fig. 5 The features after the kernel function operation. The data presents five distinct linear categorizations after M-RVM kernel processing

own length and set the regional mutation rate to 0.01 , some associated SNVs were planted in the preset elevated region and the background mutation rate was set to 0.0001 . About one fourth inserted fragments of the complex indel came from nearby regions [27]. We set the read length to $100 \mathrm{bps}$, the distribution of insert sizes to follow the normal distribution of the 500 bps mean and $15 \mathrm{bps}$ standard deviation, and the sequence error rate of reads sampling was considered as 0.005 .

The main evaluation index of the performance of the method are the accuracy and the number of relevant vectors, and are mainly affected by the kernel parameter. The accuracy refers to the ratio of the number of samples correctly classified by the classifier to the total number of samples for a given test dataset, and the relevant vector refers to the nonzero parameter corresponding point, which reflects the characteristics of the training data onto the reason that most of the parameters of the posterior distribution tend to zero and has nothing to do with forecast [29]. We first randomly selected five groups of coverage and copy number for parametric adjustment experiments and analyzed the influence of parameters on the accuracy of the method as shown in Fig. 6. The vertical axis represents the accuracy and the number of relevant vector, while the horizontal axis represents the parameter. The results showed that when the kernel parameter was 0.7 (or 10), the accuracy was the highest with the least relevant vectors, which provide some enlightenment for parameter selection.

\section{Comparing the performance on simulated dataset}

To evaluate the performance of the proposed method comprehensively, we chose three existing methods to compare: 1) obtain the CNV region through the Facets [23] and then estimated the genotypes by $20 \%-80 \%$ rule (correct the interval by the copy-number), the snp.vcf.gz file required for the Facets was downloaded at https://www.ncbi.nlm.nih.gov/variation/docs/human variation_vcf/. 2) GATK [24], default setting with HaplotypeCaller. 3) Gindel [13]. We compared the result at different copy numbers, and for each copy number configuration, we changed the coverage from $5 \times$ to $20 \times$. The accuracy of each experiment were listed in Table 2 and the third column represented the result from the proposed approach, the accuracy of Gindel, Facets, GATK were listed at column 4,5,6, respectively. Each result was an average of five repeated experiments.

The result demonstrated that the accuracy of our method was stable above $83 \%( \pm 2 \%)$ while the classification accuracies of M-SVM, Facets and GATK were very low and unstable. The average of $M-S V M$ was $45 \%$ and the range was about 30\%, the mean of Facets was 52.79\% and the range of it was $10.70 \%$, of GATK were $63.8 \%$ and $57.95 \%$. Specifically, as the coverage increased, the accuracies of these methods showed an increasing trend, and the methods decreased slightly as the copy number increased, which were all consistent with the theoretical principle. When the coverage and copy number changed, our method showed stable adaptability, strong robustness and maintained a high level. Furthermore, we visualized the variation of the relevant vectors in the iteration process as shown in Fig. 7, in which the horizontal axis represented the number of iterations, and the vertical axis represented the number of relevant vectors. The relevant vectors in the iterative learning process were obviously reduced after about 200 iterations, which showed that our 


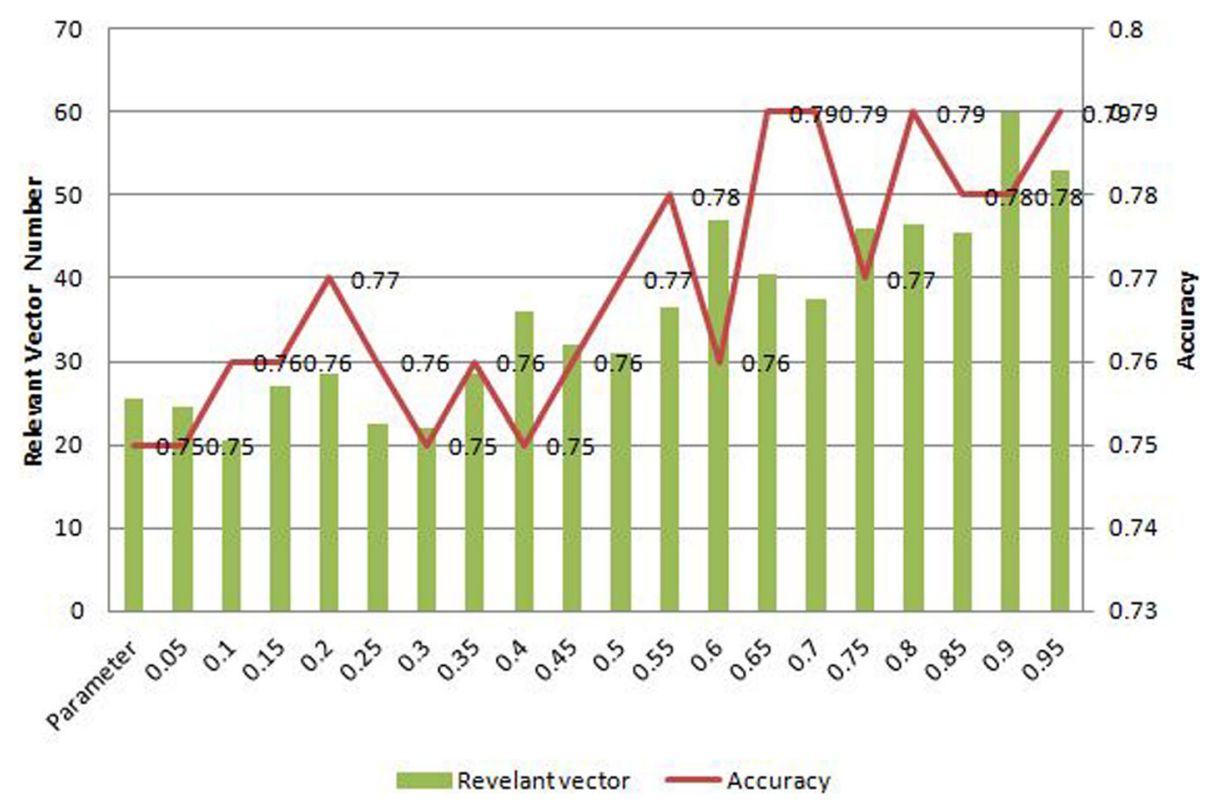

Fig. 6 Kernel parameter selection. The vertical axis represents the accuracy and the number of relevant vector, while the horizontal axis represents the parameter. The results showed that when the kernel parameter is 0.7 (or 10), the accuracy is the highest and the number of relevant vectors is the least, which provide some enlightenment for parameter selection

model only needed a small number of relevant vectors and the data sparsity was reduced with the increase in iteration steps by the M-RVM approach. Consequently, our method could achieve higher model sparsity and shorter diagnosis time, the computational complexity was low on new sample input data diagnosis.

\section{Comparing the performance on real dataset}

We obtained nine set of sequencing data from the Gene+ public database provided by Henan Cancer Hospital. All of the nine sets were the targeted sequencing reads with Gene+ 1021 panel. All the clinical information were removed, patients were numbered by a random target and all the germline variants were also removed before we obtained the data. The raw data has been already processed on the public data base, following the pipeline which the raw sequence read was mapped by $B W A-0.7 .5 a$ [25] and GATK MUTect, CNVkit were used to detect the true structural variation information.

We obtained the input SAM file and VCF file of the algorithm by processing the offline data and compared the proposed method with popular machine learning framework SVM, Nä̈ve Bayes, BP Neural Network and Lanrange-SVM with OVO multiple classification as shown in Tables 3, 4 and Fig. 8. Because the popular method Gindel selected the discordant pair reads, split mapped read, read depth as features and was a binary classification method based on the SVM framework, we compared our method with multi-classification SVM and extra tested the Gindel features with the machine learning frameworks. The results showed that the proposed method can adapt well on real data and the experiment results were even better than simulation results because of the large sample size and high coverage of

Table 2 Performance comparison on simulated datasets

\begin{tabular}{|c|c|c|c|c|c|}
\hline Copy Number & Coverage & $\begin{array}{l}\text { The } \\
\text { proposed } \\
\text { method }\end{array}$ & Gindel & Facets & GATK \\
\hline \multirow{4}{*}{2} & $5 x$ & 0.8100 & 0.3054 & 0.4710 & 0.5328 \\
\hline & $10 x$ & 0.8353 & 0.5342 & 0.5125 & 0.8157 \\
\hline & $15 x$ & 0.8477 & 0.5728 & 0.5125 & 0.7697 \\
\hline & $20 x$ & 0.8510 & 0.6175 & 0.5150 & 0.7039 \\
\hline \multirow{4}{*}{3} & $5 x$ & 0.8142 & 0.4137 & 0.4713 & 0.7368 \\
\hline & $10 x$ & 0.8195 & 0.4205 & 0.4912 & 0.4539 \\
\hline & $15 x$ & 0.8168 & 0.4273 & 0.5124 & 0.8157 \\
\hline & $20 x$ & 0.8195 & 0.4296 & 0.4625 & 0.8355 \\
\hline \multirow{4}{*}{4} & $5 x$ & 0.8234 & 0.4296 & 0.5467 & 0.2560 \\
\hline & $10 x$ & 0.8247 & 0.4201 & 0.5626 & 0.5942 \\
\hline & $15 x$ & 0.8234 & 0.4268 & 0.5672 & 0.6714 \\
\hline & $20 x$ & 0.8395 & 0.4336 & 0.5695 & 0.7101 \\
\hline \multirow{4}{*}{5} & $5 x$ & 0.8313 & 0.4796 & 0.5573 & 0.3125 \\
\hline & $10 x$ & 0.8358 & 0.4877 & 0.5630 & 0.6057 \\
\hline & $15 x$ & 0.8356 & 0.4905 & 0.5645 & 0.6153 \\
\hline & $20 x$ & 0.8413 & 0.4592 & 0.5680 & 0.7788 \\
\hline
\end{tabular}




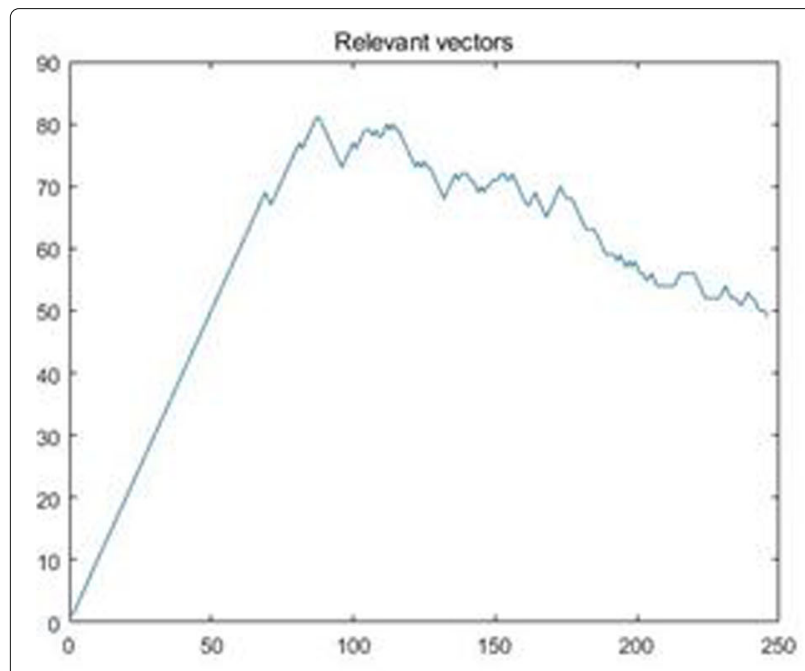

(a)

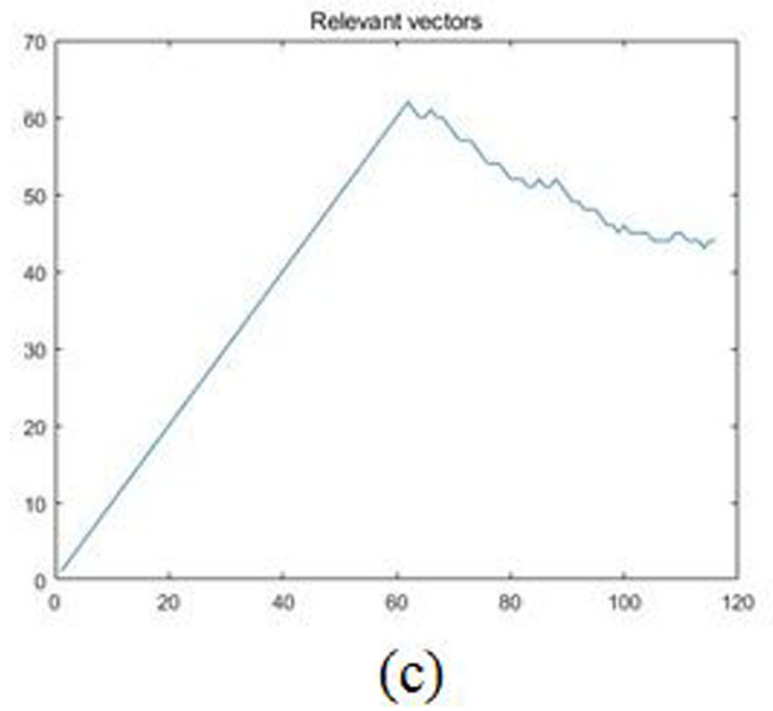

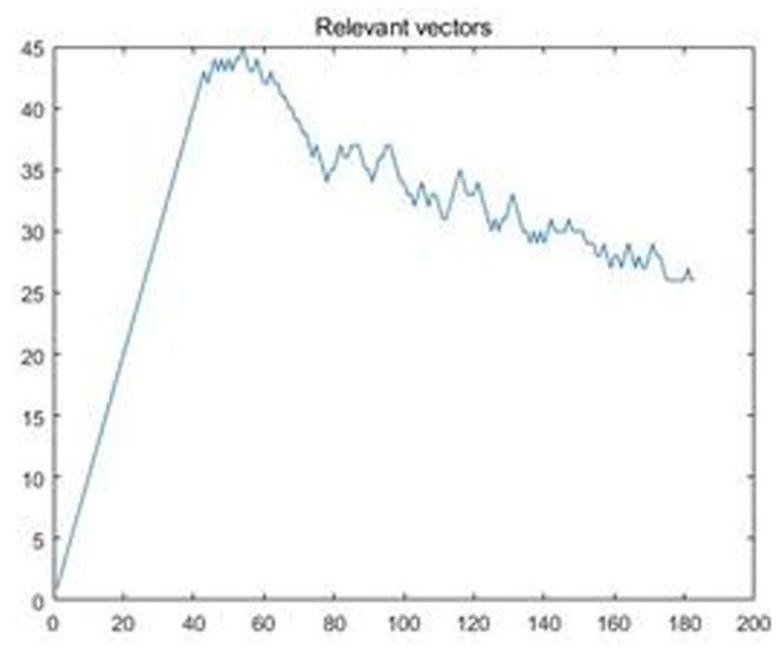

(b)

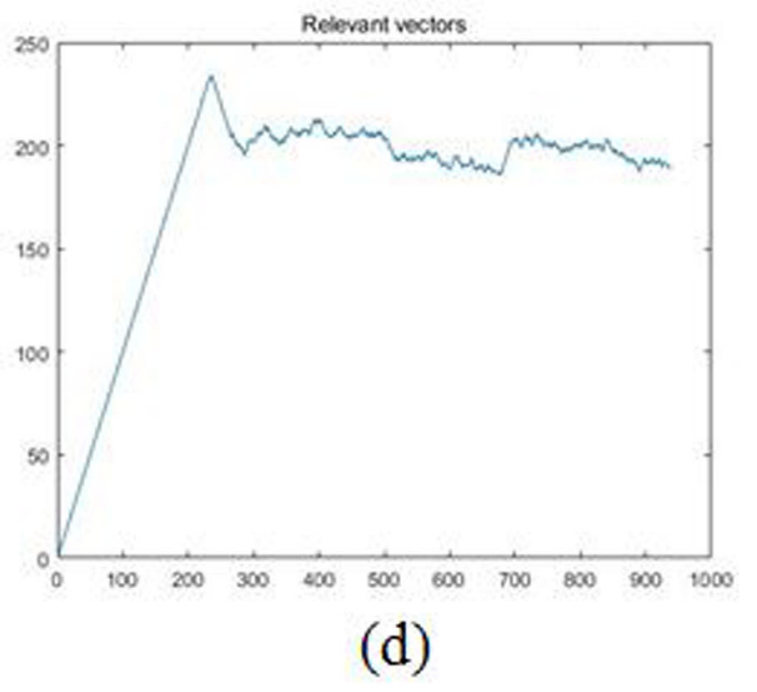

Fig. 7 Relevant vectors during the simulation dataset training process. The horizontal axis represents the number of iterations, and the vertical axis represents the number of relevant vectors. Figure $\mathbf{a}$ - $\mathbf{d}$ are four randomly records in multiple experiments

the real datasets. Compared with the popular algorithms, the average of our method accuracy was $88.20 \%( \pm 15 \%)$, while the average of $M-S V M$ was $79.17 \%( \pm 20 \%)$, of Nä̈ve Bayes was $75.85 \%( \pm 40 \%)$, of BP Neural Network was $83.67 \%( \pm 16 \%)$, of Lanrange-SVM with OVO multiple classification was $68.43 \%( \pm 28 \%)$, of Gindel features + $M-S V M$ was $72.31 \%( \pm 26 \%)$, of Gindel features + Naïve Bayes was $68.40 \%( \pm 34 \%)$, of Gindel features + BP Neural Network was $76.35 \%( \pm 22 \%)$, and of Gindel features + Lanrange-SVM with OVO multiple classification was $75.57 \%( \pm 23 \%)$, which indicated that our method maintained higher accuracy and stability. We also visualized the change of the relevant vectors in the iteration as shown in Fig. 9, which confirmed that our method maintained high model sparsity and short diagnosis time. These indicated that our method has the advantages of accuracy and computation, and can be well applied to clinical practice.

\section{Discussion}

\section{Performance tests with considering the low complexity} region

There is a research topic in the genome called the low complexity region (LCR), which contains one or more genes, pseudogenes, gene fragments, retroviral sequences and gene regulatory regions, usually located in telomeres and telomeres. Its repeats create ambiguities in alignment 
Table 3 Performance comparison on real datasets

\begin{tabular}{|c|c|c|c|c|c|c|c|}
\hline & Coverage & InsertSize & The proposed method & G-Features+M-SVM & G-Features+NB & M-SVM & $N B$ \\
\hline 1 & $472 x$ & 216 & 0.8875 & 0.9851 & 0.9777 & 0.9880 & 0.9777 \\
\hline 2 & $396 x$ & 213 & 0.8917 & 0.9098 & 0.9031 & 0.9469 & 0.9317 \\
\hline 3 & $394 x$ & 223 & 0.9083 & 0.6265 & 0.5513 & 0.7493 & 0.6766 \\
\hline 4 & $291 \times$ & 175 & 0.9082 & 0.6403 & 0.6839 & 0.7089 & 0.8582 \\
\hline 5 & $448 x$ & 214 & 0.9000 & 0.6299 & 0.7254 & 0.7444 & 0.6886 \\
\hline 6 & $460 x$ & 210 & 0.9167 & 0.5881 & 0.3415 & 0.6502 & 0.3589 \\
\hline 7 & $402 x$ & 202 & 0.7258 & 0.4603 & 0.4632 & 0.6361 & 0.6217 \\
\hline 8 & $422 x$ & 207 & 0.8917 & 0.7444 & 0.7439 & 0.7660 & 0.7469 \\
\hline 9 & $369 x$ & 215 & 0.9083 & 0.9234 & 0.7667 & 0.9336 & 0.9669 \\
\hline \multicolumn{2}{|c|}{ Average } & & 0.8820 & 0.7230 & 0.6840 & 0.7914 & 0.7585 \\
\hline \multicolumn{2}{|c|}{ Range } & & 0.1909 & 0.5248 & 0.6362 & 0.3519 & 0.6188 \\
\hline
\end{tabular}

In practice, we adopted the multi-classification support vector machine (M-SVM) as a plus version because the Gindel is a binary classification method based on support vector machine (SVM), it will treat the other three genotypes as classification errors and lead to low accuracy when applied directly

and in genome assembly, which, in turn, can produce errors when interpreting results. Repeat that is sufficiently divergent do not present problems, the LCR defined a repeat as a sequence that is at least $100 \mathrm{bps}$ in length, occurs two or more times in the genome and exhibits $>97 \%$ identity to at least one other copy of itself. This definition excludes many repetitive sequences, but it includes those present the principal computational challenges [30].

To better test the performance of the proposed method, we tested the method on the simulated dataset considering the low complexity region. We downloaded the bed file of LCR from ENCODE Project (Encyclopedia of DNA Elements) website, and inserted the recorded LCR information into the corresponding region fragments of chromosome hg19 and constructed reference containing LCR. The simulation data which meet the requirements of the existing popular literature [31] was constructed through the structural variation and CNV planting. The results were shown in the Table 5, where the average of the experiment was $81.51 \%$, which indicated that our method can effectively deal with the LCR impact.

\section{Result discussion and further study plan}

We try to explore the loss of accuracy of our method, which can be discussed from three aspects. First, the reason why that the classification accuracy of the simulation data is lower than the real data; Second, the reason for the accuracy loss of the real data; Third, the reason why the accuracy of real data fluctuates. Based on the experiment results, the accuracy of the simulation data is $83 \%$ on average, and the mean accuracy of the real data is $88.20 \%$. We analyzed the data in detail and found that the reason for this phenomenon is that the sample size and the coverage of the simulation data is small, which we set as 300 and 5 $\sim 20 \times$. In comparison, the scale of real data is thousands of times of simulation data, and the coverage is dozens of

Table 4 Performance comparison on real datasets-2

\begin{tabular}{llllll}
\hline & The proposed method & $B P$ & G-Features+BP & SVM with OVO & G-Feature+SVM with OVO \\
\hline 1 & 0.8875 & 0.9929 & 0.9868 & 0.9279 & 0.9897 \\
2 & 0.8917 & 0.9677 & 0.9122 & 0.8753 & 0.8910 \\
3 & 0.8270 & 0.6190 & 0.5653 & 0.7699 \\
4 & 0.9251 & 0.7983 & 0.7131 & 0.8018 \\
5 & 0.9082 & 0.7870 & 0.6417 & 0.4540 & 0.6057 \\
6 & 0.9000 & 0.7324 & 0.6566 & 0.4017 & 0.5884 \\
7 & 0.9167 & 0.6752 & 0.5807 & 0.6455 & 0.6016 \\
8 & 0.7258 & 0.7724 & 0.7513 & 0.6131 & 0.6302 \\
9 & 0.8917 & 0.8513 & 0.9250 & 0.9633 & 0.9230 \\
Average & 0.9083 & 0.8367 & 0.7635 & 0.6843 & 0.7557 \\
Range & 0.8820 & 0.3177 & 0.4061 & 0.5616 & 0.4013 \\
\hline
\end{tabular}




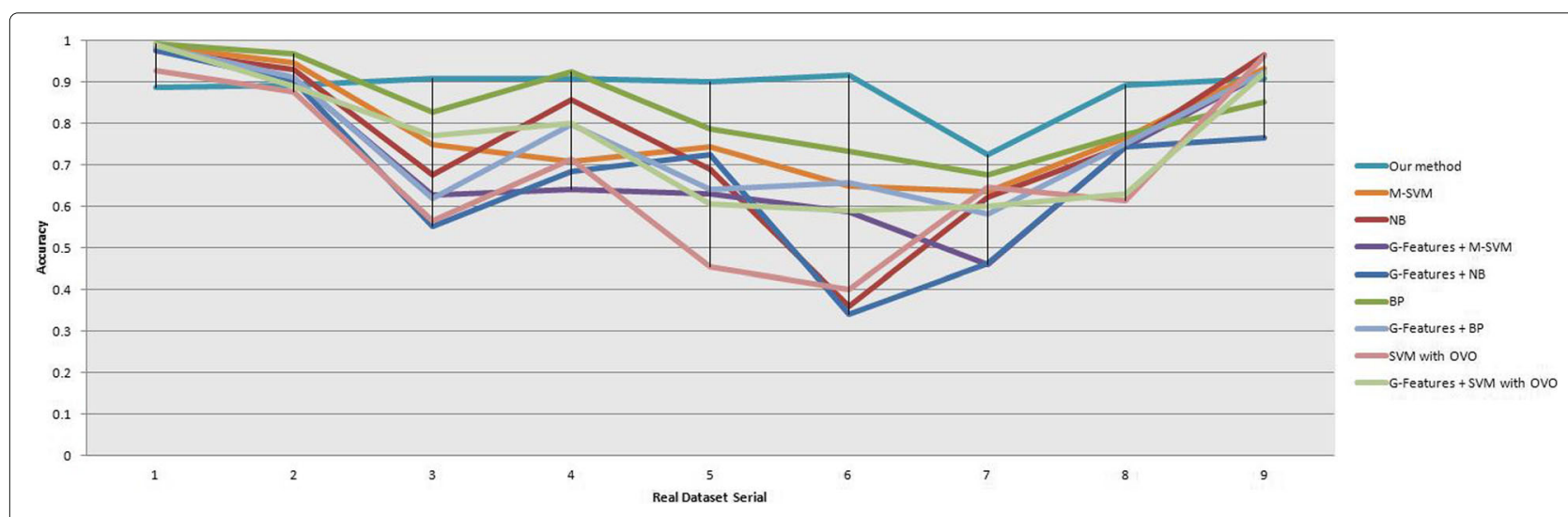

Fig. 8 Methods comparison results on real dataset. The horizontal axis represents the real dataset serial, and the vertical axis represents the accuracy

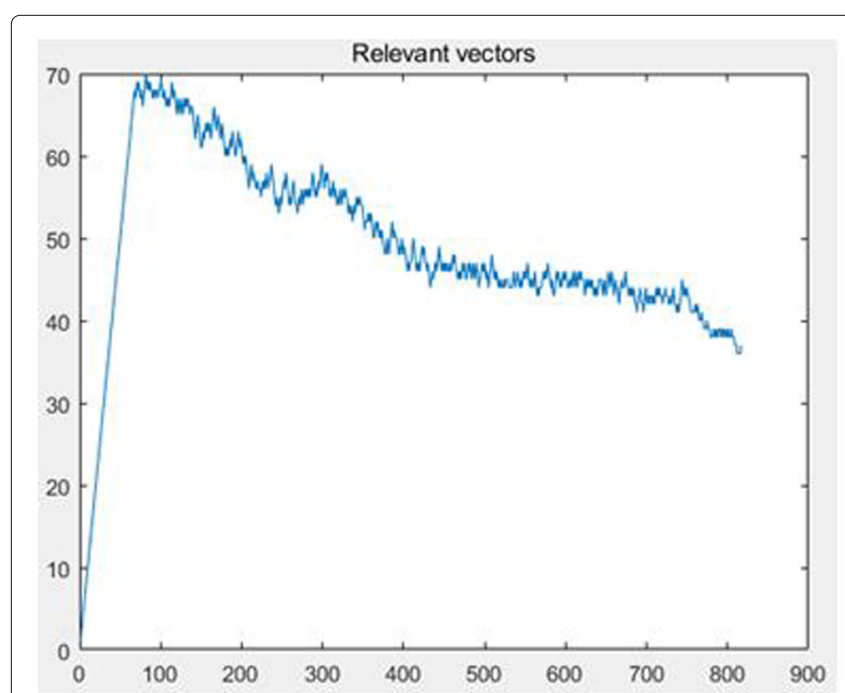

(a)

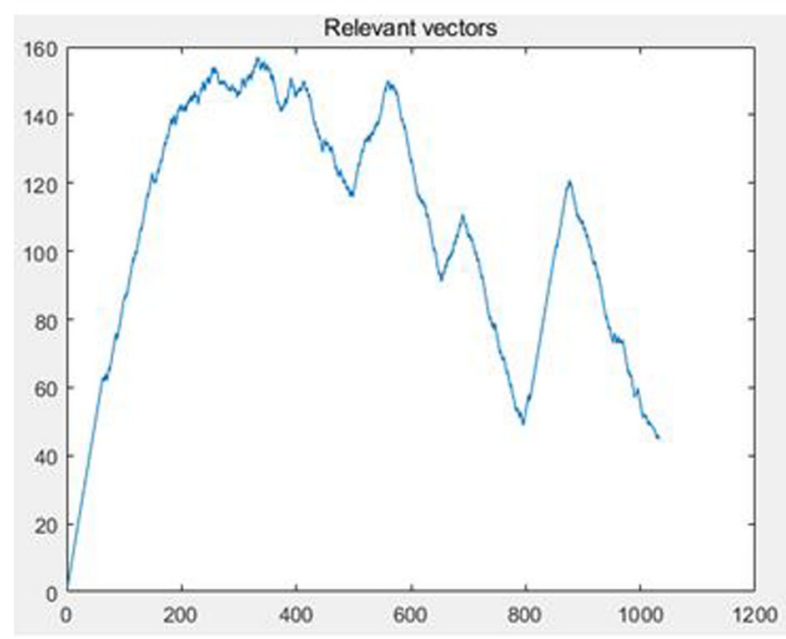

(c)

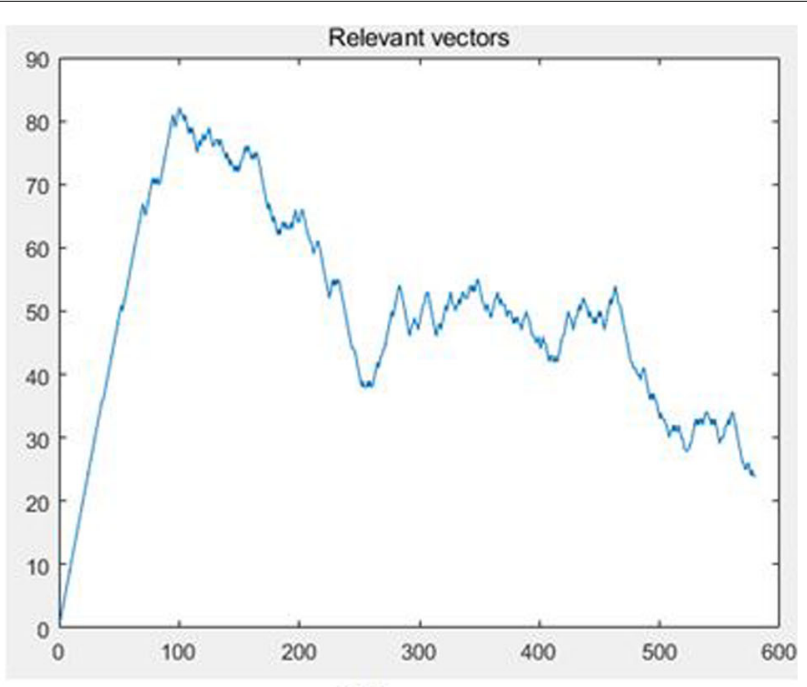

(b)

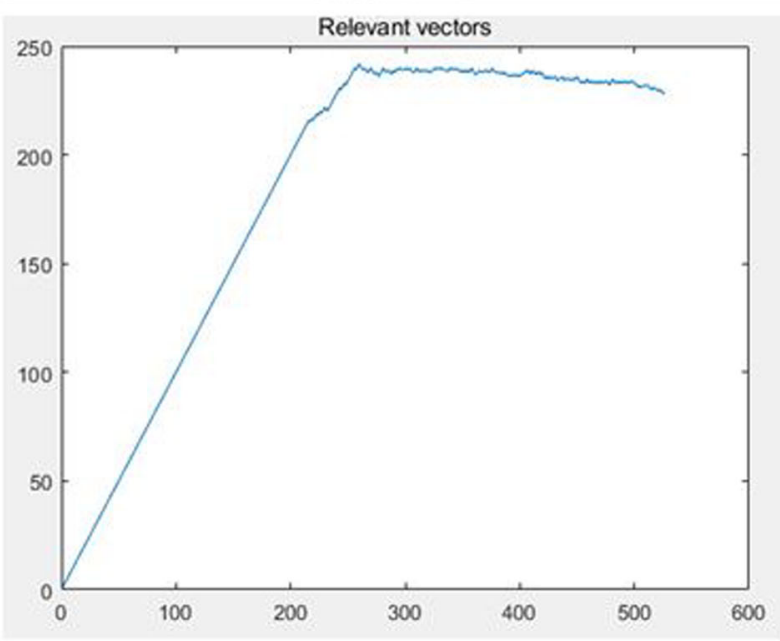

(d)

Fig. 9 Relevant vectors during the real dataset training process. The horizontal axis represents the number of iterations, and the vertical axis represents the number of relevant vectors. Figure $\mathbf{a}$ - $\mathbf{d}$ are four randomly records in multiple experiments 
Table 5 Performance test considering of the low complexity region

\begin{tabular}{llll}
\hline Copy Number & Coverage & Accuracy & Parameter number \\
\hline \multirow{2}{*}{5} & $5 \times$ & 0.7800 & 8 \\
& $10 \times$ & 0.8153 & 14 \\
& $15 \times$ & 0.8277 & 20 \\
& $20 \times$ & 0.8310 & 35 \\
3 & $5 \times$ & 0.7942 & 10 \\
& $10 \times$ & 0.8177 & 15 \\
& $15 \times$ & 0.8168 & 22 \\
& $20 \times$ & 0.8195 & 37 \\
& $5 \times$ & 0.7934 & 12 \\
& $10 \times$ & 0.8147 & 24 \\
& $15 \times$ & 0.8285 & 32 \\
& $20 \times$ & 0.8295 & 37 \\
5 & $5 \times$ & 0.8113 & 14 \\
& $10 \times$ & 0.8258 & 17 \\
& $15 \times$ & 0.8256 & 24 \\
& $20 \times$ & 0.8113 & 37 \\
\hline
\end{tabular}

times of simulation data, which has better classification accuracy and model training advantage consequently.

Secondly, we try to explain the accuracy loss of the real datasets. On the one hand, the accuracy of the machine learning model is not expected to be $100 \%$. The more classification categories, the higher the probability of accuracy loss will be. Moreover, we observed the output probability matrix of the model and found that there are a small number of samples have the equal calculated probabilities of five genotypes, our method directly considered these samples as error classifications. We counted the number of the sample of equal probability results in the real datasets as shown in Table 6, and found that the samples of equal probability were $1 \%$ of the total samples and the range was $2.04 \%$. We set the equal probability samples as invalid

Table 6 Result discussion

\begin{tabular}{lllll}
\hline & Accuracy & Sample capacity & Invalid sample & $\begin{array}{l}\text { Accuracy of } \\
\text { valid sample }\end{array}$ \\
\hline 1 & 0.8875 & 960 & 23 & 0.9093 \\
2 & 0.8917 & 4800 & 35 & 0.8983 \\
3 & 0.9083 & 2624 & 23 & 0.9163 \\
4 & 0.9082 & 3990 & 27 & 0.9144 \\
5 & 0.9000 & 6810 & 30 & 0.9040 \\
6 & 0.9167 & 5470 & 25 & 0.9210 \\
7 & 0.7258 & 1047 & 23 & 0.7421 \\
8 & 0.8917 & 2024 & 21 & 0.9011 \\
9 & 0.9083 & 6950 & 25 & 0.9116 \\
\hline
\end{tabular}

samples and recalculated the ratio of the correct classified samples of all the valid samples. The mean accuracy was $0.89 \%$ higher than the original accuracy, and the range growth was $1.85 \%$.

In addition, we also found some problems worthy of further study. When dealing with real data, there were not many samples of genotype markers for copy number loci (which is the reason that we only did real data experiments for nine patients). One reason is that the sequence company lack of this awareness, for the mechanism of individual copy number is relatively clear, and other unclear ones are not necessary for labeling. Another reason is costing considerations. Motivated by these, we want to explore the use of semi-supervised machine learning framework to learn only a small number of labeled data and train a general model for classification. The idea is being explored in another article.

\section{Conclusion}

In this article, we focused on the genotyping of structural variations with copy number variant, and proposed a machine learning method based on M-RVM. CNV is widely exists in cancer genome, which causes the misjudgment of structural variation genotyping by existing methods and greatly reduces the accuracy of processing cancer data. The correctly distinguish the position of $\mathrm{CNV}$ from the structural variation genotypes is necessary. Accordingly, we transformed the problem of genotyping into a multi-classification problem and 15 features were carefully selected as input on the basis of observation and practice. Based on the data analysis of features, we chose M-RVM framework, which can efficiently deal with the problem of low-dimensional linear inseparability, achieve efficient classification results and output the result of genotyping with the greatest possibility. We tested the performance of this method and compared it with existing popular genotype method Gindel, GATK, Facets and four commonly used machine learning methods: $S V M$, Nä̈ve Bayes, BP Neural Network and Lanrange-SVM with OVO multiple classification. The results showed that the proposed method significantly improved the accuracy of structural variations genotyping and the mean recognition rate of this method was obviously higher than other classification methods under the same conditions. In conclusion, our method is stable, reliable, robust, useful for genotyping and downstream operation, and has good response to coverage and copy number, which anticipates a wider usage in cancer clinical sequence.

\footnotetext{
Abbreviations

CNV: Copy number variant; SVs: Structural variations; VAF: Variant allelic frequency; SAM: Standard alignment/map; BAM: Binary alignment/map; VCF: Variant call format; SNV: Single nucleotide variant; M-RVM: Multiclass relevance vector machine; bp: Base pair; M-SVM: Multiclass support vector machine; BP Neural Network: Back propagation neural network; NB: Naïve Bayes; OVO: One-versus-one; LCR: Low complexity region
} 


\section{Acknowledgements}

The authors would like to thank the conference organizers of the 15th International Symposium on Bioinformatics Research and Applications (ISBRA 2019). We also would like to thank the reviewers for their valuable comments and suggestions, which guide us to improve the work and manuscript.

\section{About this supplement}

This article has been published as part of BMC Medical Genomics Volume 13 Supplement 6, 2020: Selected articles from the 15th International Symposium on Bioinformatics Research and Applications (ISBRA-19): medical genomics. The full contents of the supplement are available online at https:// bmcmedgenomics.biomedcentral.com/articles/supplements/volume-13supplement-6.

\section{Authors' contributions}

JYW and XPZ conducted this research. TZ, XYZ and ZMZ designed the computational approach. algorithms and the pipeline. TZ applied the experiments on both the simulation and real datasets. XY and HLL collected and provided the real datasets. TZ and JYW wrote this manuscript. All authors read and approved the final version of this manuscript.

\section{Authors' information}

Tian Zheng is currently a Ph.D. candidate in the School of Computer Science and Technology, Xi'an Jiaotong University, China. Her current research interests include bioinformatics, machine learning and data mining. Xiaoyan Zhu is an Associate Professor in the School of Computer Science and Technology, Xi'an Jiaotong University, China. Her current research interests include machine learning, data mining, software engineering and bioinformatics. Xuanping Zhang is a Professor in the School of Computer Science and Technology, Xi'an Jiaotong University, China. His current research interests include articial intelligence, data mining and bioinformatics. Zhongmeng Zhao is a Professor in the School of Computer Science and Technology, Xi'an Jiaotong University, China. His current research interests include information processing technology, database and data mining and bioinformatics. Xin Yi is currently the Chairman and Chief Technology Officer of Geneplus-Beijing. His current research interests include precision medical applications on tumor diagnosis and treatment, sequencing products, liquid biopsy for early-stage cancer detection. Jiayin Wang is a Professor in the School of Computer Science and Technology, Xi'an Jiaotong University. His current research interests include cancer genomics and bioinformatics. Hongle Li is vice director of the Department of Molecular Pathology, Henan Cancer Hospital, the Affiliated Cancer Hospital of Zhengzhou University. His current research interests include tumor diagnosis and treatment, tumor recurrence and metastasis.

\section{Funding}

Publication costs were funded by the National Science Foundation of China (Grant No: 31701150) and the Fundamental Research Funds for the Central Universities (CXTD2017003). The funding bodies had no role in: the design of the study; collection, analysis, and interpretation of data; or in the writing of the manuscript.

\section{Availability of data and materials}

The datasets used and analysed during the current study are available from the corresponding author on reasonable request. The source codes have been uploaded at https://github.com/TrinaZ/Mixgenotype for academic use only.

\section{Ethics approval and consent to participate}

Not applicable.

\section{Consent for publication}

Not applicable.

\section{Competing interests}

Xin $Y_{i}$ is supported by GenePlus Beijing Institute. The authors declare no other competing interests.

\section{Author details}

${ }^{1}$ School of Computer Science and Technology, Xi'an Jiaotong University, Xi'an 710049, China. ${ }^{2}$ Geneplus-Beijing, Beijing 102206, China. ${ }^{3}$ Department of Molecular Pathology, Henan Cancer Hospital, The Affiliated Cancer Hospital of Zhengzhou University, Zhengzhou 450003, China.
Received: 4 May 2020 Accepted: 25 May 2020 Published: 27 August 2020

\section{References}

1. Li P, Guo M, Wang C, Liu X, Zou Q. An overview of SNP interactions in genome-wide association studies. Brief Funct Genomics. 2015;14(2): $143-55$.

2. Alkan C, Coe BP, Eichler EE. Genome structural variation discovery and genotyping. Nat Rev Genet. 2011;12:363-76.

3. Zheng T, Li Y, Geng Y, Zhao Z, Zhang X, Wang J. Abstract 5294 CIMerge: A machine learning approach for merging and genotyping complex indel calls from NGS data. Cancer Res. 2018;78:5294.

4. Walve R, Salmela L, Mäkinen V. Variant genotyping with gap filling. Plos ONE. 2017;12(9):e0184608.

5. Walters RG, Coin LJM, Aimo R, Smith AJD, Moustafa ES, Sebastien J, Paul E, T Nu E, Anna-Liisa H, Jaana L. Rare genomic structural variants in complex disease: lessons from the replication of associations with obesity. Plos ONE. 2013;8(3):e58048.

6. Cristina E, Gelsomina F, Cristina R, Aurora D, José B, Jordi GM, María Luisa GG, Ana Isabel LS, Almudena L, Monforte AJ. SNP genotyping in melons: genetic variation, population structure, and linkage disequilibrium. Theor Appl Genet. 2013;126(5):1285-303.

7. Jonathan $S$, Lakshmi $B$, Jennifer $T$, Joan $A$, Janet $Y, P$ ? R L, Susanne $M$, Hillary M, Megan W, Maoyen C. Large-scale copy number polymorphism in the human genome. Science. 2004;305(5683):525-8.

8. Leung DYM, Nelson HS, Szefler SJ, Busse WW. Drug hypersensitivity. J Allergy Clin Immunol. 2004;113(3):372.

9. Luqing $S$, Kai L, Zheng Y. Resistance mutations against $\mathrm{HCV}$ protease inhibitors and antiviral drug design. Curr Pharm Des. 2014;20(5):694-703.

10. Ye K, Wang J, Jayasinghe R, Lameijer EW, Mcmichael JF, Ning J, Mclellan MD, Xie M, Song C, Yellapantula V. Systematic Discovery of Complex Indels in Human Cancers. Nat Med. 2016;22:97-104.

11. Vikas B, Ondrej L. A probabilistic method for the detection and genotyping of small indels from population-scale sequence data. Bioinformatics. 2011;27(15):2047-53.

12. Marschall T, Hajirasouliha I, Schönhuth A. MATE-CLEVER: Mendelian-inheritance-aware discovery and genotyping of midsize and long indels. Bioinformatics. 2013;29(24):3143-50.

13. Chu C, Zhang J, Wu Y. GINDEL: accurate genotype calling of insertions and deletions from low coverage population sequence reads. Plos ONE. 2013;9(11):e113324.

14. Zheng T, Li Y, Geng Y, Zhao Z, Zhang X, Xiao X, Wang J. CIGenotyper: A Machine Learning Approach for Genotyping Complex Indel Calls. In: Bioinformatics and Biomedical Engineering. Springer International Publishing; 2018. p. 473-85. https://doi.org/10.1007/978-3-319-787237_41.

15. Agúndez JAG, Gallardo L, Ledesma MC, Lozano L, Rodriguezlescure A, Pontes JC, Iglesiasmoreno MC, Poch J, Ladero JM, Benítez J. Functionally Active Duplications of the CYP2D6 Gene Are More Prevalent among Larynx and Lung Cancer Patients. Oncology. 2001;61:59-63.

16. Zhang X, Xu M, Wang Y, Gao A, Zhao Z, Huang Y, Xiao X, Wang J. An graph-based algorithm for prioritizing cancer susceptibility genes from gene fusion data. In: Oncology; 2017. p. 2204-10. https://doi.org/10. 1109/bibm.2017.8218000.

17. Iranmanesh SM, Guo NL. Integrated DNA Copy Number and Gene Expression Regulatory Network Analysis of Non-small Cell Lung Cancer Metastasis. Cancer Inform. 2014;13(Suppl 5):13-23.

18. Buckland PR. Polymorphically duplicated genes: their relevance to phenotypic variation in humans. Ann Med. 2003;35(5):308-15.

19. Conrad DF, Pinto D, Redon R, Feuk L, Gokcumen O, Zhang Y, Aerts J, Andrews TD, Barnes C, Campbell P, Fitzgerald T, Hu M, Ihm CH, Kristiansson K, Macarthur DG, Macdonald JR, Onyiah I, Pang AWC, Robson S, Stirrups K, Valsesia A, Walter K, Wei J, Wellcome TCCC, Tyler-Smith C, Carter NP, Lee C, Scherer SW, Hurles ME. Origins Hand functional impact of copy number variation in the human genome. Nature. 2010;464(7289):704-12.

20. Lu C, Xie M, Wendl MC, Wang J, Mclellan MD, Leiserson MDM, Huang K, Wyczalkowski MA, Jayasinghe R, Banerjee T. Patterns and functional implications of rare germline variants across 12 cancer types. Nat Commun. 2015;6:10086. 
21. The Cancer, Genome Atlas Research Network, Huang K, Mashl R, Wu Y, Ritter D, Wang J, Oh C, Paczkowska M, Reynolds S, Wyczalkowski M, Oak N, Scott A, Krassowski M, Cherniack A, Houlahan K, Jayasinghe R, Wang L, Zhou D, Liu D, Cao S, Kim Y, Koire A, McMichael J, Hucthagowder V, Kim T, Hahn A, Wang C, McLellan M, Al-Mulla F, Johnson K, CaesarJohnson S, Demchok J, Felau I, Kasapi M, Ferguson M, Hutter C, Sofia H, Tarnuzzer R, Wang Z, Yang L, Zenklusen J, Zhang J, Chudamani S, Liu J, Lolla L, Naresh R, Pihl T, Sun Q, Wan Y, Wu Y, Zaren H. Pathogenic Germline Variants in 10,389 Adult Cancers. Cell. 2018;173(2):355-70.e14.

22. Hai Y, Weishi Y, Velculescu VE, Bert V, Kinzler KW. Allelic variation in human gene expression. Science. 2002;297(5584):1143.

23. Shen $R$, Seshan VE. FACETS: allele-specific copy number and clonal heterogeneity analysis tool for high-throughput DNA sequencing. Nucleic Acids Res. 2016;44(16):e131.

24. Mckenna A, Hanna M, Banks E, Sivachenko A, Cibulskis K, Kernytsky A, Garimella K, Altshuler D, Gabriel S, Daly M. The Genome Analysis Toolkit: A MapReduce framework for analyzing next-generation DNA sequencing data. Genome Res. 2010;20(9):1297-303.

25. Li H. Aligning sequence reads, clone sequences and assembly contigs with BWA-MEM. ArXiv. 2013;1303:

26. Simonsanchez J, Scholz S, Fung HC, Matarin M, Hernandez D, Gibbs JR, Britton A, de Vrieze FW, Peckham E, Gwinnhardy K. Genome-wide SNP assay reveals structural genomic variation, extended homozygosity and cell-line induced alterations in normal individuals. Hum Mol Genet. 2007;16:1-14.

27. Geng Y, Zhao Z, Xu M, Zhang X, Xiao X, Wang J. TNSim: A Tumor Sequencing Data Simulator for Incorporating Clonality Information; 2018, pp. 371-82. https://doi.org/10.1007/978-3-319-95933-7_45.

28. Tipping ME. Sparse Bayesian Learning and the Relevance Vector Machine. J Mach Learn Res. 2001;1:211-44.

29. Hao X, Tianzhen W, Tianhao T, Benbouzid MEH. A PCA-mRVM fault diagnosis strategy and its application in CHMLIS. In: IECON 2014 - 40th Annual Conference of the IEEE Industrial Electronics Society; 2014. p. 1124-30.

30. Treangen TJ, Salzberg SL. Repetitive DNA and next-generation sequencing: computational challenges and solutions. Nat Rev Genet. 2011;13:36-46.

31. Li H. Towards Better Understanding of Artifacts in Variant Calling from High-Coverage Samples. Bioinformatics. 2014;30:. https://doi.org/10. 1093/bioinformatics/btu356.

\section{Publisher's Note}

Springer Nature remains neutral with regard to jurisdictional claims in published maps and institutional affiliations.

Ready to submit your research? Choose BMC and benefit from:

- fast, convenient online submission

- thorough peer review by experienced researchers in your field

- rapid publication on acceptance

- support for research data, including large and complex data types

- gold Open Access which fosters wider collaboration and increased citations

- maximum visibility for your research: over $100 \mathrm{M}$ website views per year

At BMC, research is always in progress.

Learn more biomedcentral.com/submissions 